\title{
Superficial Thrombophlebitis caused by Extensively Drug-resistant Salmonella Enterica Serovar Typhi
}

\author{
Anam Imtiaz, Irfan Ali Mirza, Qanita Fahim and Nargis Sabir \\ Department of Microbiology, Combined Military Hospital, Lahore, Pakistan
}

\begin{abstract}
Salmonella enterica serovar typhi causes one of the most common blood stream infections, the typhoid fever. However, it can cause pyogenic infections involving different sites as well. Extensively drug resistant (XDR) strains of Salmonella typhi are resistant to all first line anti-typhoidal drugs (chloramphenicol, ampicillin and trimethoprim-sulfamethoxazole) as well as ciprofloxacin and ceftriaxone. XDR-strains were first reported from Pakistan in 2016, and since then the strains have been spreading. These XDR Salmonella cases not only pose a therapeutic challenge but also predispose to complications as a result of prolonged illness and delayed treatment. Here, we report a case of superficial thrombophlebitis at intravenous cannula site in a 49-year male, who was being treated for XDR-typhoid fever. To the best of our knowledge, thrombophlebitis of a superficial vein is an unusual complication of Salmonella typhi, not previously reported in literature.
\end{abstract}

Key Words: Bacteremia, Thrombophlebitis, Extensively drug-resistant, Typhoid fever, Salmonella typhi.

How to cite this article: Imtiaz A, Mirza IA, Fahim Q, Sabir N. Superficial Thrombophlebitis caused by Extensively Drug-resistant Salmonella Enterica Serovar Typhi. J Coll Physicians Surg Pak 2020; 30(11):1217-1219.

\section{INTRODUCTION}

Salmonella enetrica serovar typhi causes one of the most common blood stream infections, the typhoid fever. However, it can cause pyogenic infections as well. Soft tissue abscesses, wound infections, genital and hepatobiliary tract as well as vascular infections have all been reported. ${ }^{1}$ In the antibiotic era, complications related to typhoid fever became less frequent because of early institution of effective antibiotic therapy. But with the emergence of extensively drug-resistant (XDR) strains that are resistant to all first line anti-typhoidal drugs (chloramphenicol, ampicillin and trimethoprim-sulfamethoxazole) as well as ciprofloxacin and ceftriaxone, the complications may again become prevalent. All recommended treatments are ineffective against these strains. A delayed treatment predisposes the patients of XDR-typhoid to prolonged illness and complications. ${ }^{2,3}$

Here, we report a case of superficial thrombophlebitis at intravenous cannula site in a patient of XDR-typhoid fever.

Correspondence to: Dr. Anam Imtiaz, Department of Microbiology, Combined Military Hospital, Lahore,

Pakistan

E-mail: anam.igb@gmail.com

Received: September 12, 2019; Revised: October 31, 2019;

Accepted: November 02, 2019

DOI: https://doi.org/10.29271/jcpsp.2020.11.1217

\section{CASE REPORT}

A 49-year male, known diabetic, reported to the medical outpatient department (OPD) with history of high grade intermittent fever for the past one week. At presentation, the patient had a fever of $101^{\circ} \mathrm{F}$. Baseline investigations were done and a paired blood culture sample was taken for culture and sensitivity testing (Tablel).

The patient was admitted in the medical ward. Intravenous ceftriaxone at a dose of $2 \mathrm{~g}$ daily was started as empirical antibiotic. Patient continued to have fever spikes despite starting the antibiotic therapy. The blood culture yielded the growth of XDR Salmonella enterica subspecies enterica serovar typhi (resistant to ampicillin, trimethoprim-sulfamethoxazole, chloramphenicol, ciprofloxacin and ceftriaxone). The culture and antimicrobial susceptibility report was conveyed to the treating physician. The antibiotic was changed to injection meropenem, $1 \mathrm{~g}$, thrice daily and oral azithromycin, $500 \mathrm{mg}$, once daily. On the $4^{\text {th }}$ day of admission, redness and swelling were noted at the intravenous cannula site over left forearm. The cannula was removed and re-sited on the right forearm. By $7^{\text {th }}$ day, the swelling and pain increased over left forearm, a thickened venous cord was palpable at the area. Patient again had fever spikes. Doppler ultrasound of left upper limb showed subcutaneous fluid collection with thrombophlebitis involving basilic vein and venae comitantes of brachial artery along with cellulitis of the surrounding skin. The infected thrombosed vein was surgically removed along with debridement of the surrounding skin. The pus collection was drained intraoperatively and sent to microbiology departmentforcultureand sensi- 
tivity testing. The pus was reddish brown in color and thick in consistency. Gram staining of the specimen showed numerous pus cells and gram negative bacilli. The specimen was inoculated on Sheep blood agar and MacConkey agar (Oxoid, UK). On next day, there was growth of flat, spreading colonies that were non-lactose fermenting and were oxidase negative. The isolate identification was done on the basis of biochemical reactions on API 20 E (bioMerieux, France) and antimicrobial susceptibility testing was performed by Kirby Bauer disk diffusion method using the Clinical and Laboratory Standards Institute (CLSI) guidelines. ${ }^{4}$ The isolate was identified as Salmonella enterica serovartyphi, that was XDR and only susceptible to meropenem (MIC 0.64) and azithromycin (MIC 0.25). The patient's antibiotics were continued for 12 more days in-hospital. His fever and swelling over the arm settled with treatment. He was advised oral azithromycin, 500 mg, once daily for 10 days on discharge. On follow-up visit after two weeks, the patient did not report any fever or swelling at the site.

Table I: Antimicrobial susceptibility pattern of the Salmonella typhi isolated from paired blood culture.

\begin{tabular}{|l|l|l|}
\hline \multicolumn{3}{|l|}{ Blood culture: Salmonella enterica serovar typhi } \\
\hline \multirow{4}{*}{$\begin{array}{l}\text { Antimicrobial } \\
\text { susceptibility } \\
\text { testing (AST) }\end{array}$} & Ampicillin (10 ug) & Resistant \\
\cline { 2 - 3 } & Chloramphenicol (10 ug) & Resistant \\
\cline { 2 - 3 } & Trimethoprim-sulfamethoxazole & Resistant \\
\cline { 2 - 3 } & Ciprofloxacin & Resistant \\
\cline { 2 - 3 } & Ceftriaxone (5ug) & Resistant \\
\cline { 2 - 3 } & Azithromycin (15 ug) & $\begin{array}{c}\text { Sensitive } \\
\text { (MIC }=0.25)\end{array}$ \\
\cline { 2 - 3 } & Meropenem (10 ug) & $\begin{array}{c}\text { Sensitive } \\
(\text { MIC }=0.64)\end{array}$ \\
\hline
\end{tabular}

\section{DISCUSSION}

Pakistan is currently facing the largest epidemic of drug resistant typhoid fever. Cases of XDR-Salmonella typhi were first reported from Hyderabad, Pakistan in 2016; and since then, there have been reports of similar cases throughout the country. ${ }^{5}$ The authors report an unusual complication of typhoid fever that was noted during the ongoing outbreak of XDR Salmonellatyphi in Pakistan. The patient developed thrombophlebitis of basilic vein along with cellulitis of the surrounding tissue where the intravenous cannula was placed for administration of antibiotics.

The clinical spectrum of Salmonella infections may at times vary from the usual blood stream infection and gastroenteritis to different extra-intestinal focal forms. Various complications can occur that involve central nervous system, pulmonary system, musculoskeletal, hepatobiliary and genitourinary systems. ${ }^{6}$ Salmonella infections cause bacteremia associated with a widespread inflammatory response. Thrombus development in Salmonella infections may be related to tissue inflammation triggered by the innate, TLR4-dependent inflammatory cascade. ${ }^{7}$ Cases of infections of arterial aneurysms, grafts and deep veins due to non-typhoidal Salmonella species have been reported in literature; however, to the best of ourknowledge, no case of thrombophlebitis involving superficial veins secondary to typhoidal Salmonellae has been reported earliar in literature. In the cases of vascular infections previously reported, associated bacteremia was established in only few cases. ${ }^{8-10}$ The Salmonella species identified in these cases were $S$. typhimurium and $S$. enteritidis. One case of cutaneous vasculitis with pancreatitis and splenic abscess, secondary to Salmonella typhi bacteremia, has been previously reported. Diabetes mellitus has been reported as a risk factor for extra-intestinal dissemination. ${ }^{6}$ The patient in this case was a known type-2 diabetic for the past 12 years.

The isolate in the present case was XDR. There is one previously reported case of extra-intestinal infection by XDR-Salmonella in literature, which was a case of breast abscess in a pregnant lady reported from Karachi, Pakistan. The infection was treated with a combination of meropenem and azithromycin as was our patient. Meropenem is not a recommended treatment for typhoid fever. It was, however, used successfully for the treatment of XDR-typhoid cases in the outbreak in Pakistan. ${ }^{11}$

The extra-intestinal manifestations of Salmonellae infections should be kept in mind, especially in areas where the disease is endemic. The bacterium can disseminate to virtually all internal organ systems once the bacteremia is established. Vigilance in early identification of these extra-intestinal foci is, therefore, imperative. It is also emphasised that there is an urgent need to look for newer treatment options for typhoidal Salmonellae and breakpoints for susceptibility testing of meropenem against Salmonellaeshould be introduced in guidelines.

\section{PATIENT'S CONSENT:}

Informed consent was obtained from the patient to publish the data concerning this case.

\section{CONFLICT OF INTEREST:}

The authors declared no conflict of interest.

\section{AUTHORS' CONTRIBUTION:}

Al, IAM, QF, NS: Identification and follow-up of case, drafting manuscriptand its revision.

\section{REFERENCES}

1. Lalitha MK, John R. Unusual manifestations of salmonellosis-a surgical problem. Q J Med 1994; 87(5): 301-9.

2. Yousafzai TM, Qamar FN, Shakoor S, Saleem K, Lohana H, Karim S, et al. Ceftriaxone-resistant Salmonella typhi outbreak in Hyderabad city of Sindh, Pakistan: High time for the introduction of typhoid conjugate vaccine. Clin Infect Dis 2019; 68(Suppl_1):S16-S21. doi: 10.1093/cid/ ciy877.

3. Parry CM, Ribeiro I, Walia K, Rupali P, Baker S, Basnyat B. Multidrug resistant enteric fever in South Asia: Unmet medical needs and opportunities. BMJ 2019; 364:k5322. doi: 10.1136/bmj.k5322.

4. Clinical and laboratory standards institute. Performance standards for antimicrobial susceptibility testing; 26 ed. CLSI supplement M100S. Clinical and laboratory standards institute, Wayne, PA 2016. 
5. Typhoid fever: Islamic Republic of Pakistan. Disease outbreak news. WHO; 2018 Dec http://www.who.int/ csr/don/ 27-december-2018-typhoid-pakistan/en/

6. Huang DB, DuPont HL. Problem pathogens: Extra-intestinal complications of salmonella enterica serotype typhi infection. Lancet Infect Dis 2005; 5(6):341-8. doi: 10.1016/S1473-3099(05)70138-9.

7. Hitchcock JR, Cook CN, Bobat S, Ross EA, Flores-Langarica A, Lowe $\mathrm{KL}$. Inflammation drives thrombosis after salmonella infection via CLEC-2 on platelets. J Clin Invest 2015; 125(12):4429-46. doi: 10.1172/JCl79070.

8. Trairatvorakul P, Sriphojanart S, Sathapatayavongs B. Abdominal aortic aneurysms infected with Salmonella:
Problems of treatment. J Vasc Surg 1990; 12(1):16-9. doi: 10.1067/mva.1990.19944.

9. Hakim S, Davila F, Amin M, Hader I, Cappell MS. Infectious aortitis: A life-threatening endovascular complication of nontyphoidal salmonella bacteremia. Case Rep Med 2018; 2018:6845617. doi: 10.1155/2018/6845617.

10. Prakash PS, Nomellini V, Kaplan LJ. Lower extremity salmonella abscess with vascular thrombosis and wall destruction. Surg Infect Case Rep 2016; 1(1):79-810. doi.org/10.1089/crsi.2016.0017.

11. Zahra NM, Satti L, Hanif F, Nadeem S. Unilateral breast abscess by extremely drug resistant Salmonella enterica serovar Typhi: First case report from Pakistan. JCDR 2019; 13(4):1-22. 